\title{
Prevalence, distribution and evolutionary significance of the IS629 insertion element in the stepwise emergence of Escherichia coli 0157:H7
}

\author{
Lydia V Rump ${ }^{1,2^{*}}$, Markus Fischer ${ }^{2}$ and Narjol Gonzalez-Escalona ${ }^{1}$
}

\begin{abstract}
Background: Insertion elements (IS) are known to play an important role in the evolution and genomic diversification of Escherichia coli 0157:H7 lineages. In particular, IS629 has been found in multiple copies in the E. coli O157:H7 genome and is one of the most prevalent IS in this serotype. It was recently shown that the lack of O157 antigen expression in two O rough E. coli O157:H7 strains was due to IS629 insertions at 2 different locations in the gne gene that is essential for the $O$ antigen biosynthesis.

Results: The comparison of 4 E. coli O157:H7 genome and plasmid sequences showed numerous IS629 insertion sites, although not uniformly distributed among strains. Comparison of IS629s found in O157:H7 and O55:H7 showed the presence of at least three different IS629 sub-types. O157:H7 strains carry IS629 elements sub-type I and III whereby the ancestral O55:H7 carries sub-type II. Analysis of strains selected from various clonal groups defined on the E. coli 0157:H7 stepwise evolution model showed that IS629 was not observed in sorbitol fermenting $\mathrm{O} 157$ (SFO157) clones that are on a divergent pathway in the emergence of O157:H7. This suggests that the absence of IS629 in SFO157 strains probably occurred during the divergence of this lineage, albeit it remains uncertain if it contributed, in part, to their divergence from other closely related strains.

Conclusions: The highly variable genomic locations of IS629 in O157:H7 strains of the A6 clonal complex indicates that this insertion element probably played an important role in genome plasticity and in the divergence of O157: $\mathrm{H7}$ lineages.
\end{abstract}

\section{Background}

Enterohemorrhagic Escherichia coli (EHEC) of serotype O157:H7 has been implicated in foodborne illnesses worldwide. It frequently causes large outbreaks of severe enteric infections including bloody diarrhoea, hemorrhagic colitis (HC) and haemolytic uremic syndrome (HUS) $[1,2]$. This serotype constitutively expresses the somatic (O) 157 and flagellar (H) 7 antigens, thus, these traits are used extensively in clinical settings to identify this highly pathogenic serotype [1]. However some O157:H7 strains, although being genotipically $\mathrm{O} 157$ or $\mathrm{H} 7$ do not express either of those antigens [3,4]. According to the latest CDC report, E. coli O157:H7 infections affect

\footnotetext{
* Correspondence: Lydiarump@gmail.com

'Division of Microbiology, Center for Food Safety and Applied Nutrition, Food and Drug Administration, 5100 Paint Branch Parkway, College Park, MD 20740, USA

Full list of author information is available at the end of the article
}

thousands of people every year accounting for $0.7 \%, 4 \%$ and $1.5 \%$, of illnesses, hospitalizations and deaths, respectively of the total U.S. foodborne diseases caused by all known foodborne pathogens [5].

Previously, we characterized two potentially pathogenic $\mathrm{O}$ rough: $\mathrm{H} 7$ strains that did not express the $\mathrm{O} 157$ antigen $[4,6]$ but belonged to the most common O157: $\mathrm{H} 7$ clonal type. The $\mathrm{O}$ rough phenotype was found to be due to two independent IS629 insertions in the gne gene that encodes for an epimerase enzyme essential for synthesis of an oligosaccharide subunit in the $\mathrm{O}$ antigen. Of the IS elements identified in O157 strains, IS629 elements are the most prevalent in this serotype and have been confirmed to very actively transpose in O157 genomes [7]. The presence of O-rough strains of this serotype in food and clinical samples is of concern as they cannot be detected serologically in assays routinely used to test for $\mathrm{O} 157: \mathrm{H} 7$ [3]. 
The occurrence of other atypical O157:H7 strains due to IS629 insertions therefore, might be more common than anticipated. It is generally assumed that IS elements play important roles in bacterial genome evolution and in some cases are known contributors to adaptation and improved fitness [7]. The acquisition or loss of mobile genetic elements, like IS elements, may differ between strains of a particular bacterial species [8]. IS insertion and IS-mediated deletions have been shown to generate phenotypic diversity among closely related O157 strains [7]. It has been shown that O157 is a highly diverse group and a major factor that effects this diversity are prophages [7]. However, in addition to prophages, IS629 also appears to be a major contributor to genomic diversification of $\mathrm{O} 157$ strains. Therefore, it is questionable how much influence IS629 had on the evolution of O157:H7, or how much importance IS629 has to changes in virulence in this bacterium.

It has been proposed in an evolutionary model previously that highly pathogenic enterohemorrhagic E. coli (EHEC) O157:H7 arose from its ancestor enteropathogenic E. coli (EPEC) O55:H7 (SOR+ and GUD+) through sequential acquisition of virulence, phenotypic traits, and serotypic change $\left(\mathrm{A} 1\left(\operatorname{stx}^{-}\right) / \mathrm{A} 2(\operatorname{st} x 2)\right.$ in Figure 1A) [9-11]. After the somatic antigen change from $\mathrm{O} 55$ to $\mathrm{O} 157$ gave rise to an intermediary (A3) which has not yet been isolated, two separate O157

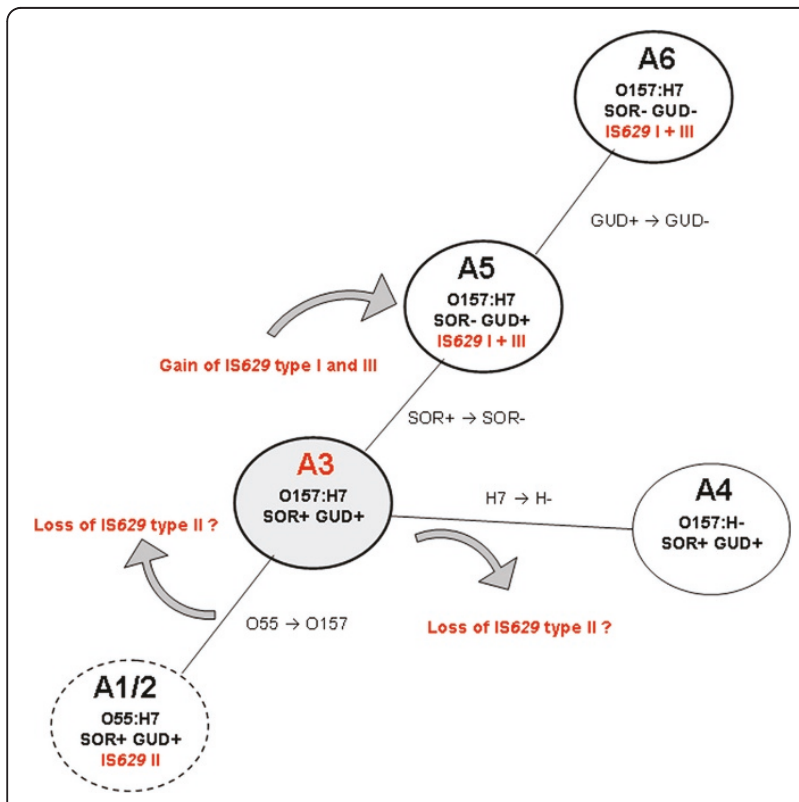

Figure 1 Stepwise evolutionary model for E. coli 0157:H7 from ancestral 055:H7 [11]. In red letters are the possible events

happening and where they occurred during the stepwise evolution. The circle in gray represents an intermediary A3 CC, which has not yet been isolated. SOR - sorbitol fermentation [if (+) fermenting, if (-) non-fermenting or slow fermenting]. GUD - $\beta$-D-glucuronidase activity. clonal complexes evolved, splitting into two diverged clonal groups. One of these groups was composed of sorbitol fermenting (SF) non-motile O157:NM strains containing plasmid pSFO157 (A4) (SOR+, GUD+). The other was composed of non-sorbitol fermenting (NSF) O157:H7 strains containing plasmid pO157 (A5) (SOR-, GUD+). The latter, by a mutational inactivation of the uidA gene, lost its $\beta$-glucuronidase activity which is the most typical O157:H7 phenotype at present (A6) [11] These A6 strains have spread geographically into disparate locales and now account for most of the diseases caused by EHEC [12].

IS629 seems to play an important role in the diversification of closely related strains, specifically O157:H7 [7]. In the present study, we examined the prevalence of IS629 in a panel of $E$. coli strains, including ancestral and atypical strains associated with the stepwise emergence of E. coli O157:H7 to determine the prevalence of IS629 and its impact on the transitional steps that gave rise to today's highly pathogenic E. coli O157:H7.

\section{Results}

\section{IS629 prevalence in E. coli 0157:H7 genomes}

The IS629 sequence, recently found to be inserted into the gne gene in E. coli O rough:H7 (MA6 and CB7326) $[4,13]$, was used for a BLAST analysis of the genomes of 4 E. coli O157:H7 strains belonging to A6 CC (EDL933, Sakai, EC4115 and TW14359) and one O55: H7 strain (CB9615) (Additional file 1, Table S1). The BLAST analysis for IS629 showed the presence of between 22 and 25 copies in each strain along with their corresponding plasmid (Table 1). Strains Sakai and EDL933 shared 13 of those IS629 on the chromosome and three on their pO157 plasmids. Strains EC4115 and TW14359 had 17 IS629 on the chromosome and four on their pO157 plasmid in common. The analysis of the recently released $E$. coli $\mathrm{O} 55: \mathrm{H} 7$ genome strain CB9615 [14] allowed for identification of one IS629 with an internal $86 \mathrm{bp}$ deletion on the chromosome and an IS629 in its corresponding pO55 plasmid. Neither the $\mathrm{O} 55$ genomic (located on the chromosome backbone) nor the pO55 plasmid IS629 insertion sites were present in other O157:H7 strains. The absence of the pO55 IS629 insertion site in O157: H7 strains was expected since they do not carry the pO55 plasmid. However, lack of the genomic O55 IS629 insertion site in O157:H7 strains is interesting as these strains are known to be closely related [14]. Contrary to what was observed for plasmids pO157 and pO55, IS629 was absent in plasmid pSFO157 (E. coli O157:H- strain 439-89). However, a 66 bp sequence identical to IS629 was observed in the plasmid which could be a remnant of IS629. No genomic sequence is available for an $0157: \mathrm{H}$ - strain at this time, thus, this 
Table 1 Prevalence of IS629 elements in each strain (chromosomes and plasmids) and number of shared IS629

\begin{tabular}{|c|c|c|c|c|c|c|c|c|}
\hline & \multirow[t]{2}{*}{ Strain } & \multirow[t]{2}{*}{ Serotype } & \multirow[t]{2}{*}{ IS629 Sites } & \multicolumn{5}{|c|}{ In common with strain } \\
\hline & & & & Sakai & EDL933 & EC4115 & TW14359 & CB9615 \\
\hline \multicolumn{9}{|c|}{ Chromosomes } \\
\hline & Sakai & O157:H7 & 19 & - & 13 & 9 & 9 & 0 \\
\hline & EDL933 & O157:H7 & 21 & 13 & - & 6 & 6 & 0 \\
\hline & EC4115 & O157:H7 & 19 & 9 & 6 & - & 17 & 0 \\
\hline & TW14359 & O157:H7 & 21 & 9 & 6 & 17 & - & 0 \\
\hline & CB9615 & O55:H7 & 1 & 0 & 0 & 0 & 0 & - \\
\hline \multirow[t]{7}{*}{ Plasmids } & & & & p0157 Sakai & p0157 EDL933 & p0157 EC4115 & p0157 TW14359 & p055 CB9615 \\
\hline & p0157 Sakai & O157:H7 & 3 & - & 3 & 3 & 3 & 0 \\
\hline & p0157 EDL933 & O157:H7 & 3 & 3 & - & 3 & 3 & 0 \\
\hline & p0157 EC4115 & O157:H7 & 4 & 3 & 3 & - & 4 & 0 \\
\hline & p0157 TW14359 & O157:H7 & 4 & 3 & 3 & 4 & - & 0 \\
\hline & pSFO157 & O157:H- & 0 & 0 & 0 & 0 & 0 & 0 \\
\hline & p055 CB9615 & O55:H7 & $1 \mathrm{tr}^{*}$ & 0 & 0 & 0 & 0 & - \\
\hline
\end{tabular}

$\operatorname{tr}^{*}$ - truncated.

strain could not be investigated for the presence of IS629.

IS629 target site specificity ("hot spots") on chromosomes and plasmids of four E. coli 0157:H7 strains

The majority of IS629 elements were located on prophages or prophage-like elements (62\%) ("strainspecific-loops", S-loops in Sakai [15]). 28\% of IS629 locations were found on the well-conserved $4.1-\mathrm{Mb}$ sequence widely regarded as the $E$. coli chromosome backbone (E. coli K-12 orthologous segment) [15] and $10 \%$ were located on the pO157 plasmid. In total, we observed 47 different IS629 insertion sites (containing complete or partial IS629) in the four E. coli chromosomes and plasmids by "in silico" analysis (Additional file 2, Table S2). Seven of 47 IS629 insertion were shared among the 4 diverged strains which suggest that they were also present in a common ancestor.

\section{IS629 presence in strains belonging to the stepwise model of emergence of $E$. coli 0157:H7}

A total of 27 E. coli strains (Table 2) belonging to the stepwise model proposed by Feng et al. (1998) were examined by PCR for the presence of IS629 using specific primers [16]. Every strain of clonal complex (CC) A6, A5, A2 and A1 carried IS629, except strain 3256-97 belonging to the ancestral CC A2 (Figure 1). Strikingly, however, was the observation that IS629 was absent in the SFO157 strains belonging to the closely related CC A4 (Figure 2). Whole genome analysis of two A4 strains (493-89 accession no. AETY00000000 and H2687 accession no. AETZ00000000) confirmed the absence of this specific IS element in SFO157 strains [17]. On the other hand, O55:H7 strain 3256-97 (AEUA00000000) carried a truncated IS629 version missing the target area for the reverse primer (IS629-insideR) located in ORFB, explaining the lack of IS629 by PCR [17]. Additionally, strains USDA5905 (A2) and TB182A (A1) as well as strain LSU-61 (A?) appear to harbor a truncated IS629 which could indicate the presence of genomic IS629 found in the O55 strain CB9615. However, since no additional ancestral strains were available for analysis, the distribution of IS629 in these groups is at present inconclusive.

IS629 distribution in strains belonging to the stepwise model of emergence of $E$. coli 0157:H7

We successfully PCR amplified 38 of the 47 observed IS629 insertion sites in the 27 O157:H7 strains analyzed (Additional file 3, Table S2). We determined presence or absence of an IS629 element as well as the IS629 target site in each strain (Additional file 1, Figure S1). In accordance with the previous finding of total absence of IS629 in SFO157, none of the A4 CC strains harbored an IS629 in any of the known IS629 insertion sites. Likewise, it was observed for A1 and A2 CC strains, indicating that the previously detected IS629 must be located in some other region of the chromosome. In A5 CC strains, only 3 of the 38 (7\%) IS629 insertion sites harbored an IS629 (Table 3). Those sites were located on the prophage Sp12, the prophage-like element SpLE1, and on the chromosomal backbone. Interestingly one of the A5 CC strains (strain 1659) did not share any of the known sites harboring IS629. The A6 CC strains shared between $6(16 \%)$ and 21 (55\%) IS629 insertions in the known sites and two of them (IS.15: Sp14 and IS.41: pO157) were present in all A6 CC strains. IS629 prevalence in the A6 strains and the distribution amongst Sp, SpLE, backbone and the pO157 plasmids did not show any 
Table 2 Serotype, sequence type, characteristics and isolation information of strains of $E$. coli used in this study

\begin{tabular}{|c|c|c|c|c|c|c|c|c|c|c|c|c|}
\hline \multirow[t]{2}{*}{ No. } & \multirow[t]{2}{*}{ Name } & \multirow[t]{2}{*}{ Other name } & \multirow[t]{2}{*}{ Serotype } & \multirow[t]{2}{*}{ stx } & \multicolumn{3}{|c|}{ Special characteristics } & \multirow[t]{2}{*}{ ST } & \multirow[t]{2}{*}{$\mathrm{CC}$} & \multirow[t]{2}{*}{ Source } & \multirow[t]{2}{*}{ Year } & \multirow[t]{2}{*}{ Reference } \\
\hline & & & & & GUD & SOR & plasmid & & & & & \\
\hline 1 & Sakai & BAA 460 & O157:H7 & 1,2 & - & - & p0157 & 66 & A6 & Japan & 1996 & NC_002695 \\
\hline 2 & EDL 933 & 700927 & O157:H7 & 1,2 & - & - & p0157 & 66 & & USA & 1982 & AE005174 \\
\hline 3 & EC 4115 & & O157:H7 & 1,2 & - & - & p0157 & 66 & & USA & 2006 & NC_011353 \\
\hline 4 & TW 14359 & & O157:H7 & 1,2 & - & - & p0157 & 66 & & USA & 2006 & CP001368 \\
\hline 5 & EDL 931 & 35150 & $\mathrm{O} 157: \mathrm{H7}$ & 1,2 & - & - & p0157 & 66 & & & & [26] \\
\hline 6 & MA6 & & O157:H7 & 2 & - & - & p0157 & 66 & & Malaysia & 1998 & {$[6]$} \\
\hline 7 & 550654 & & O157:H7 & 2 & - & - & p0157 & 66 & & USA & 2009 & \\
\hline 8 & FDA 413 & & O157:H7 & 2 & - & - & p0157 & 66 & & & & [10] \\
\hline 9 & G5101 & & O157:H7 & 1,2 & + & - & p0157 & 65 & A5 & USA & 1995 & [11] \\
\hline 10 & 1628 & & O157:H7 & 1,2 & + & - & p0157 & 65 & & & & [32] \\
\hline 11 & 1659 & & O157:H7 & 1,2 & + & - & p0157 & 65 & & & & [11] \\
\hline 12 & EC 97144 & TW 10707 & O157:H7 & 1,2 & + & + & p0157 & 65 & & Japan & 1997 & [33] \\
\hline 13 & EC 96038 & TW 10201 & O157:H7 & 1,2 & + & + & p0157 & 65 & & & & [11] \\
\hline 14 & EC 96012 & TW 10189 & O157:H7 & 1,2 & + & + & p0157 & 65 & & & & [11] \\
\hline 15 & 493-89 & & O157:H- & 2 & + & + & pSFO157 & 75 & A4 & Germany & 1989 & [11] \\
\hline 16 & $5412-89$ & & 0157:H- & 2 & + & + & pSFO157 & 75 & & Germany & 1989 & [34] \\
\hline 17 & H56929 & TW 09159 & O157:H- & 2 & + & + & pSFO157 & 76 & & Finland & 1999 & [11] \\
\hline 18 & H56909 & TW 09162 & O157:H- & 2 & + & + & pSFO157 & 76 & & Finland & 1999 & [11] \\
\hline 19 & H $1085 c$ & & O157:H- & 2 & + & + & pSFO157 & 76 & & Scotland & 2003 & [11] \\
\hline 20 & H 2687 & & 0157:H- & 2 & + & + & pSFO157 & 76 & & Scotland & 2003 & [11] \\
\hline 21 & $3256-97$ & TW 07815 & $\mathrm{O} 55: \mathrm{H7}$ & 2 & + & + & $?$ & 73 & $\mathrm{~A} 2$ & USA & 1997 & [11] \\
\hline 22 & USDA 5905 & & $\mathrm{O} 55: \mathrm{H7}$ & 2 & + & + & $?$ & 73 & & USA & 1994 & [26] \\
\hline 23 & TB 182A & TW 04062 & $\mathrm{O} 55: \mathrm{H7}$ & - & + & + & $?$ & 73 & $\mathrm{~A} 1$ & USA & 1991 & [11] \\
\hline 24 & DEC5A & & $\mathrm{O} 55: \mathrm{H7}$ & - & + & + & $?$ & 73 & & & & [11] \\
\hline 25 & LSU-61 & & O157:H7 & - & + & + & $?$ & 237 & $?$ & USA & 2001 & NC_002695 \\
\hline 26 & Sakai PF & & O157:H7 & 1,2 & - & - & p0157 & 66 & A6 & Japan & 1996 & AE005174 \\
\hline 27 & 43895 & $\begin{array}{c}\text { CDC } \\
\text { EDL } 933\end{array}$ & O157:H7 & 1,2 & - & - & p0157 & 69 & A6 & USA & 1982 & \\
\hline
\end{tabular}

stx - shiga toxin gene, GUD - $\beta$-glucuronidase activity, SOR - sorbitol fermentation, ST - sequence type as determined by a combination of seven genes http:// www.shigatox.net/stec/cgi-bin/index, CC - Clonal complex [11], ? - Unknown. Sakai PF and 43895 are strain derived after numerous subculture passages from the original Sakai and EDL933 strains, respectively.

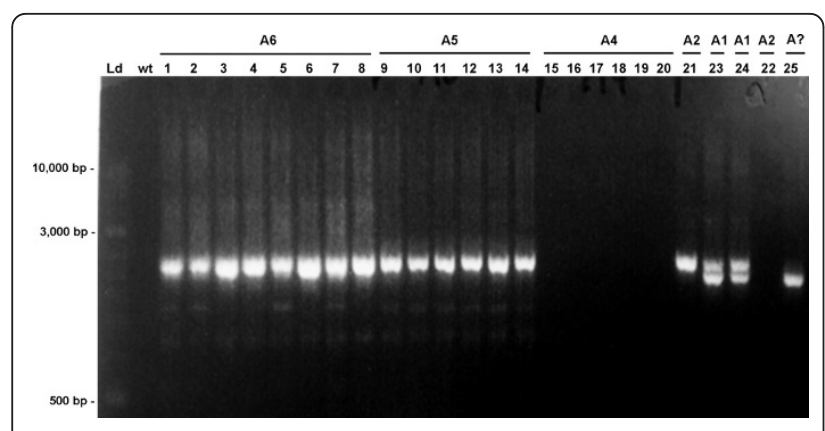

Figure 2 Gel-electrophoresis of the PCR products for IS629 presence in strains belonging to the stepwise model of emergence of $\boldsymbol{E}$. coli 0157:H7. Lanes: Ld, molecular weight ladder (Gene Ruler); wt, Blank; 1 - 25, strains numbered according to Table 2. A1-A6, Clonal complexes, A?, CC unknown. specific pattern, however it appears that IS629 transposes actively in the A6 CC.

Figure $1 \mathrm{~B}$ shows a maximum parsimony tree obtained for A5 and A6 CC strains using IS629 presence/absence in the target site and presence/absence of IS629 target site (chromosome or plasmid region) (Table 3 and Additional file 4, Table S3). Strains belonging to A1, A2, and A4 CCs were not included in this analysis because they either lack IS629 (A4) or IS629 is located in other regions on the chromosome than the ones determined for O157:H7 strains. The parsimony tree allowed to separate strains belonging to A5 from A6 strains as proposed in the stepwise model (Figure 1 and 3A) [10,12]. Furthermore, it showed the existence of high diversity among A5 and A6 CC strains similar to what has been shown by PFGE [11]. The validity of this analysis needs 
Table 3 IS629 element presence/absence in CC strains from the 0157:H7 stepwise evolutionary model

\begin{tabular}{|c|c|c|c|c|c|c|c|c|c|c|c|c|c|c|c|c|c|c|c|c|c|c|c|c|c|c|c|c|}
\hline & & & & & A6 & & & & & & & & A5 & & & & & A4 & & & & A2 & & $\mathrm{A} 1$ & & A? & & A6 \\
\hline NR & $\begin{array}{l}\text { Phage } \\
\text { Or } \\
\text { backbone }\end{array}$ & 1 & 2 & 3 & 4 & 5 & 6 & 7 & 8 & 9 & 10 & 11 & 12 & 13 & 14 & 15 & 16 & 17 & 18 & 19 & 20 & 21 & 22 & 23 & 24 & 25 & 26 & 27 \\
\hline IS.1 & Sp 4 & ND & ND & ND & ND & ND & ND & ND & ND & ND & ND & ND & ND & ND & ND & ND & ND & ND & ND & ND & ND & ND & ND & ND & ND & ND & ND & ND \\
\hline 15.2 & Sp 4 & ND & ND & ND & ND & ND & ND & ND & ND & ND & ND & ND & ND & ND & ND & ND & ND & ND & ND & ND & ND & ND & ND & ND & ND & ND & ND & ND \\
\hline IS. 3 & Sp 5 st $\times 2$ & + & - & - & - & - & - & - & - & - & - & - & - & - & - & - & - & - & - & - & - & - & - & - & - & - & + & - \\
\hline IS. 4 & SpLE 1 & - & + & - & - & - & - & + & - & - & - & - & - & - & - & - & - & - & - & - & - & - & - & - & - & - & - & - \\
\hline IS. 5 & SpLE 1 & + & + & + & + & + & - & + & - & - & - & - & - & - & - & - & - & - & - & - & - & - & - & - & - & - & + & + \\
\hline IS. 6 & SpLE 1 & - & + & - & - & - & - & + & - & - & - & - & - & - & - & - & - & - & - & - & - & - & - & - & - & - & - & - \\
\hline IS. 7 & SpLE 1 & + & + & + & + & + & - & + & + & - & - & - & - & - & - & - & - & - & - & - & - & - & - & - & - & - & + & + \\
\hline IS. 8 & Sp 8 & + & - & + & - & + & - & + & - & - & - & - & - & - & - & - & - & - & - & - & - & - & - & - & - & - & + & - \\
\hline IS. 9 & Sp 8 & - & + & - & - & - & - & - & - & - & - & - & - & - & - & - & - & - & - & - & - & - & - & - & - & - & - & + \\
\hline IS. 10 & back & + & + & - & - & + & - & + & + & - & - & - & - & - & - & - & - & - & - & - & - & - & - & - & - & - & + & + \\
\hline IS. 11 & back & + & + & - & - & + & - & - & - & - & - & - & - & - & - & - & - & - & - & - & - & - & - & - & - & - & + & + \\
\hline IS. 12 & Sp 12 & + & - & - & - & - & - & - & - & - & - & - & + & + & + & - & - & - & - & - & - & - & - & - & - & - & + & - \\
\hline IS. 13 & back & + & + & + & + & + & + & + & - & - & - & - & - & - & - & - & - & - & - & - & - & - & - & - & - & - & + & + \\
\hline IS. 14 & Sp 13 & + & + & + & + & + & + & - & + & - & - & - & - & - & - & - & - & - & - & - & - & - & - & - & - & - & + & + \\
\hline IS. 15 & Sp 14 & + & + & + & + & + & + & + & + & - & - & - & - & - & - & - & - & - & - & - & - & - & - & - & - & - & + & - \\
\hline IS.16 & SpLE 2 & ND & ND & ND & ND & ND & ND & ND & ND & ND & ND & ND & ND & ND & ND & ND & ND & ND & ND & ND & ND & ND & ND & ND & ND & ND & ND & ND \\
\hline IS. 17 & back & + & + & - & - & - & - & - & - & - & - & - & - & - & - & - & - & - & - & - & - & - & - & - & - & - & + & + \\
\hline IS. 18 & Sp 15 stx 1 & + & + & - & - & + & - & - & - & - & - & - & - & - & - & - & - & - & - & - & - & - & - & - & - & - & + & + \\
\hline IS. 19 & back & + & - & + & + & + & - & - & + & - & - & - & - & - & - & - & - & - & - & - & - & - & - & - & - & - & + & - \\
\hline IS. 20 & Sp 17 & + & - & + & + & + & - & + & + & - & - & - & - & - & - & - & - & - & - & - & - & - & - & - & - & - & + & - \\
\hline IS. 21 & SpLE3 & + & + & - & + & + & + & + & + & - & - & - & - & - & - & - & - & - & - & - & - & - & - & - & - & - & + & + \\
\hline IS.22 & back & ND & ND & ND & ND & ND & ND & $N D$ & ND & ND & ND & ND & ND & ND & ND & ND & ND & ND & ND & ND & ND & ND & $N D$ & ND & ND & ND & ND & ND \\
\hline IS. 23 & SpLE 5 & + & + & - & - & + & - & + & + & - & - & - & - & - & - & - & - & - & - & - & - & - & - & - & - & - & + & + \\
\hline IS. 24 & SpLE 1 & - & + & - & - & - & - & - & - & - & - & - & - & - & - & - & - & - & - & - & - & - & - & - & - & - & - & + \\
\hline IS. 25 & SpLE 1 & - & + & - & - & - & - & - & - & - & - & - & - & - & - & - & - & - & - & - & - & - & - & - & - & - & - & + \\
\hline 15.26 & 9330 & ND & ND & ND & ND & ND & $N D$ & ND & ND & ND & ND & $\mathrm{ND}$ & ND & ND & ND & ND & ND & ND & ND & ND & ND & ND & ND & ND & ND & ND & ND & ND \\
\hline
\end{tabular}


Table 3 IS629 element presence/absence in CC strains from the 0157:H7 stepwise evolutionary model (Continued)

\begin{tabular}{|c|c|c|c|c|c|c|c|c|c|c|c|c|c|c|c|c|c|c|c|c|c|c|c|c|c|c|c|c|}
\hline IS. 27 & SpLE 2 & - & + & - & - & - & - & - & - & - & - & - & - & - & - & - & - & - & - & - & - & - & - & - & - & - & - & + \\
\hline IS.28 & $933 Y$ & ND & ND & ND & ND & ND & ND & ND & ND & ND & ND & ND & ND & ND & ND & ND & ND & ND & ND & ND & ND & ND & ND & ND & ND & ND & ND & ND \\
\hline IS. 29 & Sp 1 & - & - & + & + & - & - & - & + & - & - & - & - & - & - & - & - & - & - & - & - & - & - & - & - & - & - & - \\
\hline IS. 30 & Sp 4 & - & - & + & - & - & - & - & - & - & - & - & - & - & - & - & - & - & - & - & - & - & - & - & - & - & - & - \\
\hline IS. 31 & Phage & - & - & + & + & - & - & - & - & + & + & - & + & + & + & - & - & - & - & - & - & - & - & - & - & - & - & - \\
\hline IS. 32 & back & - & - & + & + & - & - & - & + & - & - & - & - & - & - & - & - & - & - & - & - & - & - & - & - & - & - & - \\
\hline IS. 33 & Sp 13 & - & - & + & + & - & - & - & - & - & - & - & - & - & - & - & - & - & - & - & - & - & - & - & - & - & - & - \\
\hline IS. 34 & back & - & - & + & + & - & - & - & - & - & - & - & - & - & - & - & - & - & - & - & - & - & - & - & - & - & - & - \\
\hline IS. 35 & Sp 5 stx 2 & - & - & + & + & - & - & - & + & - & - & - & - & - & - & - & - & - & - & - & - & - & - & - & - & - & - & - \\
\hline IS.36 & back & ND & ND & ND & ND & ND & ND & ND & ND & ND & ND & ND & ND & ND & ND & ND & ND & ND & ND & ND & ND & ND & ND & $N D$ & ND & ND & ND & ND \\
\hline IS. 37 & Phage & - & - & + & + & - & - & - & - & - & - & - & - & - & - & - & - & - & - & - & - & - & - & - & - & - & + & - \\
\hline IS. 38 & back & - & - & + & + & - & - & - & - & - & - & - & - & - & - & - & - & - & - & - & - & - & - & - & - & - & - & - \\
\hline IS. 39 & (gne gene) & - & - & - & - & - & + & - & - & - & - & - & - & - & - & - & - & - & - & - & - & - & - & - & - & - & - & - \\
\hline IS. 40 & p0157 & + & - & - & - & + & - & - & - & - & - & - & - & - & - & - & - & - & - & - & - & - & - & - & - & - & + & - \\
\hline IS. 41 & p0157 & + & + & + & + & + & + & + & + & - & - & - & - & - & - & - & - & - & - & - & - & - & - & - & - & - & + & + \\
\hline IS. 42 & p0157 & - & - & + & + & - & - & - & - & - & - & - & - & - & - & - & - & - & - & - & - & - & - & - & - & - & + & + \\
\hline 15.43 & p0157 & & & & & & & & & & & & & & & & & & & & & & & & & & & \\
\hline IS. 44 & p0157 & - & - & + & + & - & - & - & - & - & - & - & - & - & - & - & - & - & - & - & - & - & - & - & - & - & - & - \\
\hline IS. 45 & p0157 & - & - & & + & - & - & - & & - & - & - & - & & - & - & - & - & - & - & - & - & & & - & - & - & \\
\hline IS. 46 & back & - & - & - & + & - & - & & & + & + & - & - & & - & - & - & - & - & - & - & - & & & - & - & - & \\
\hline IS.47 & back & ND & ND & ND & ND & ND & ND & ND & ND & ND & ND & ND & ND & ND & ND & ND & ND & $\mathrm{ND}$ & ND & $N D$ & ND & ND & ND & ND & ND & ND & ND & ND \\
\hline 15.48 & p0157 & ND & ND & ND & ND & ND & ND & ND & ND & ND & ND & ND & ND & ND & ND & ND & ND & ND & ND & ND & ND & ND & ND & ND & ND & ND & ND & ND \\
\hline
\end{tabular}

IS629 sites were numbered from 1 - 47 (NR) starting with all sites in Sakai, followed by all additional, unshared sites from EDL933, EC4115, the sites found in the plasmids and unshared sites of strain TW1435. The newly found IS629 insertion in O rough:H7 strain MA6 was numbered IS.39 [4]. A1 - A6 are strains belonging to the different clonal complexes.

$\mathrm{Sp}$ - Phage; SpLE - Phage-like element; back - backbone; ND -Not determined, primers failed to amplify the region. 


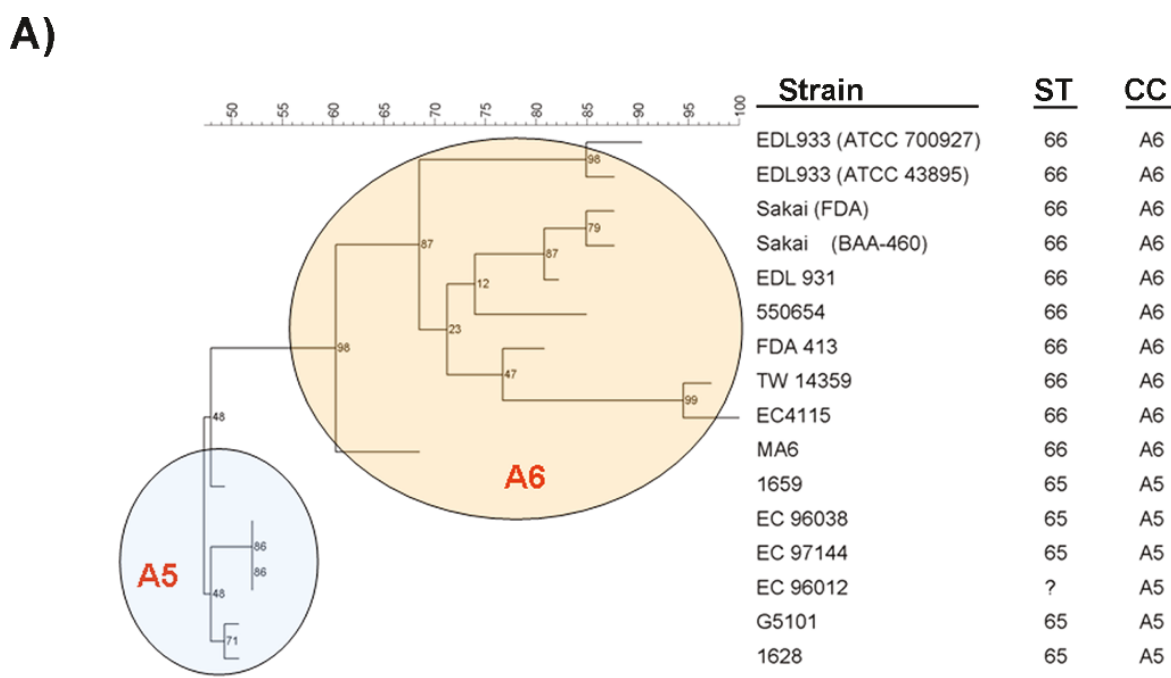

B)

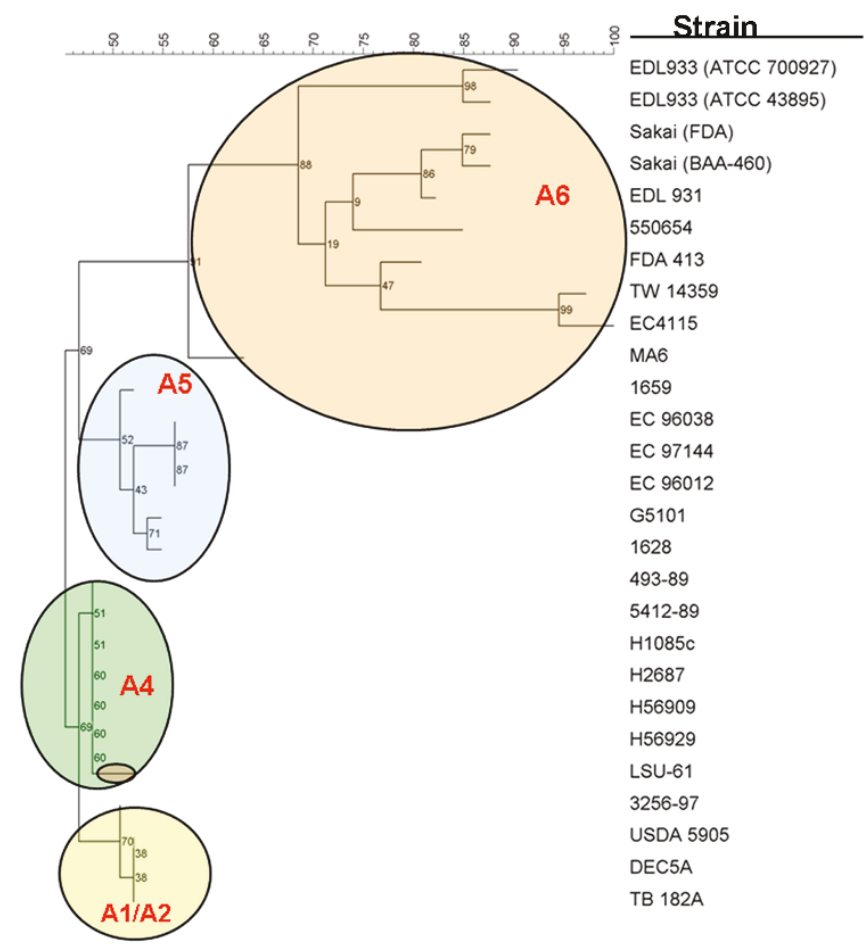

$\begin{array}{lll}\frac{\text { ST }}{66} & & \text { CC } \\ 66 & \text { A6 } \\ 66 & \text { A6 } \\ 66 & \text { A6 } \\ 66 & \text { A6 } \\ 66 & \text { A6 } \\ 66 & \text { A6 } \\ 66 & \text { A6 } \\ 66 & \text { A6 } \\ 66 & \text { A6 } \\ 66 & \text { A6 } \\ 65 & \text { A5 } \\ 65 & \text { A5 } \\ 65 & \text { A5 } \\ ? & \text { A5 } \\ 65 & \text { A5 } \\ 65 & \text { A5 } \\ 75 & \text { A4 } \\ 75 & \text { A4 } \\ 76 & \text { A4 } \\ 76 & \text { A4 } \\ 76 & \text { A4 } \\ 76 & \text { A4 } \\ 237 & \text { ? } \\ 73 & \text { A2 } \\ 73 & \text { A2 } \\ 73 & \text { A1 } \\ 73 & \text { A1 }\end{array}$

Figure 3 Evolutionary significance of IS629 in the emergence of E. coli 0157:H7. A) Maximum parsimony tree obtained using the distribution of IS629 and IS629 target sites in the 14 O157:H7 strains analyzed in the present study (Table 3 and Additional file 4, Table S3). B) Maximum parsimony tree obtained using IS629 target sites for the 27 strains analyzed in the present study (Additional file 4, Table S3). The colored ellipses mark the different CCs. CC - clonal complex; ST - sequence type.

to be explored further using more O157:H7 strains belonging to either A5 or A6 CCs. Besides using 25 different strains for the analysis, we also included additional Sakai and EDL933 strains. Sakai strains were one from ATCC (BAA-460) and the other from a personal collection (FDA). EDL933 strains were provided by ATCC whereby strain EDL933 700927 derived from
EDL933 43895. PFGE analysis showed only minimal changes between the original (ATCC) and the derived ones confirming their identity (data not shown). The analysis using the IS629 distribution also showed minimal changes in the IS629 distribution as well among the Sakai and EDL933 strains. The use of IS629 presence/ absence in specific regions has been used before to help 
detecting outbreak related strains as described by Ooka, et al (2009) and for population genetics analysis as described by Yokoyama, et al (2011), and appears to be a promising and adequate technique to distinguish closely related O157:H7 strains. However, both methodologies takes no notice of the information about the presence of the region where IS629 was inserted into. The presence/absence of a specific region in $E$. coli O157:H7 chromosomes, irrelevant of the presence of IS629, could provide additional information regarding relatedness among those strains.

IS629 insertion site prevalence in the strains belonging to the stepwise model of emergence of $E$. coli 0157:H7

PCR analysis for the presence of IS629 insertion sites showed that sites located on the chromosomal backbone structure were present in all tested strains from the different clonal complexes (Table 4 and Additional file 4). However, neither A1, A2, nor A4 CC strains harbored any IS629 in backbone IS629 insertion sites.

Contrary to what was observed in the well-conserved backbone, IS629 insertion sites in prophages and prophage-like elements in different strains were found to be highly variable (Table 5 and Additional file 4, Table S3). As seen for the backbone IS629 insertion sites, some of the phage associated IS629 insertions sites were present in A1, A2 and A4 CC strains; however they lacked IS629. Many of the IS629 sites on phages were unique to the A6 CC strains (7 of 13) suggesting that they are strain-specific. This result underscores significant differences in the presence of phage-related sequences between the strains belonging to the stepwise model of E. coli O157:H7.

The two IS629 insertions in O55 and its corresponding plasmid pO55 were observed to be present in only one ancestral A2 and both A1 CC strains (data not shown). A6, A5, and A4 CC strains as well as A2 CC strain 3256-97 (IS629-deficient) lacked the IS629 insertion site in these regions. Interestingly, strain LSU-61

Table 4 Presence of IS629 target sites on the backbone

\begin{tabular}{ccccccc}
\hline IS629 target sites & A1 & A2 & A3 & A4 & A5 & A6 \\
\hline IS.10 & $+/-$ & + & NA & + & + & $+/-$ \\
IS.11 & + & + & NA & + & + & + \\
IS.13 & + & + & NA & + & + & + \\
IS.17 & + & + & NA & + & + & + \\
IS.19 & + & + & NA & + & + & + \\
IS.32 & + & + & NA & + & + & + \\
IS.34 & + & + & NA & + & + & + \\
IS.38 & + & + & NA & + & + & + \\
IS.39 & + & + & NA & + & + & + \\
IS.46 & - & - & NA & $+/-$ & + & + \\
\hline
\end{tabular}

NA, not applicable; + presence; - absence; +/- present in some strains.
Table 5 Presence of phage or phage-like associated IS629 target sites

\begin{tabular}{ccccccc}
\hline IS629 target sites & A1 & A2 & A3 & A4 & A5 & A6 \\
\hline Sp 1 & - & - & NA & - & - & + \\
Sp 2 & + & + & NA & + & + & + \\
Sp 4 & + & + & NA & + & + & + \\
Sp 5 & - & - & NA & - & - & + \\
Sp 8 & - & - & NA & - & - & + \\
Sp 12 & - & + & NA & + & + & + \\
Sp 13 & - & - & NA & - & - & + \\
Sp 14 & - & - & NA & + & + & + \\
Sp 17 & - & - & NA & - & - & + \\
SpLE 1 & - & - & NA & - & + & + \\
SpLE 2 & - & - & NA & - & - & + \\
SpLE 3 & - & - & NA & - & - & + \\
SpLE 5 & - & - & NA & - & + & + \\
\hline
\end{tabular}

Sp - Phage; SpLE - Phage-like element; NA - not applicable; + presence; absence.

which carries multiple characteristics for O157:H7 and is thought to be ancestral to A5 CC strains (Feng et al 2007), appeared to carry the truncated genomic IS629 insertion.

Since the strains belonging to the stepwise model share variable IS629 insertion sites we reconstructed their evolutionary path using this information. A parsimony tree using the IS629 target sites presence/absence produced a tree that was nearly analogous to the proposed model of stepwise evolution for O157:H7 from ancestral O55:H7 strains [10], with A1/A2 CC strains at the base of the tree, followed by A4 CC, A5 CC and A6 $\mathrm{CC}$ strains in that order (Figure $3 \mathrm{~B}$ ).

\section{Phylogenetic analysis of IS629 elements in the four E. coli 0157:H7 and 055:H7 genomes}

The phylogenetic analysis of IS629 elements revealed that IS629 in E coli O157:H7 can be divided into three different sub-types (Figure 4). That is, IS629 of sub-type I and II differ in average $4 \%$ (> $55 \mathrm{bp}$ ) while sub-type II and III differed by $5 \%$ (> $60 \mathrm{bp}$ ). Sub-type I appears to be most closely related to those of IS1203 (IS629 isoform) found in O111:H- [18]. IS629 sub-type II appears to be most closely related to those of IS629 found in Shigella [19]. IS629 sub-type III appears to be most closely related to those of IS629 found in E. coli O26:H11 [20]. Therefore, analysis of all targeted IS629 elements showed that strains from A6 CC seem to carry both IS1203 (sub-type I) and IS629 (sub-type III) whereby the ancestral O55:H7 strain carries IS629 (sub-type II). Since IS629 sub-type II found in the ancestral O55:H7 strain is significantly different from the other two IS629 sub-types (O157:H7 strains) and sub-type II is no longer present in certain O157:H7 strains (A6 CC), these data imply that IS629 sub-type I and III were recently 


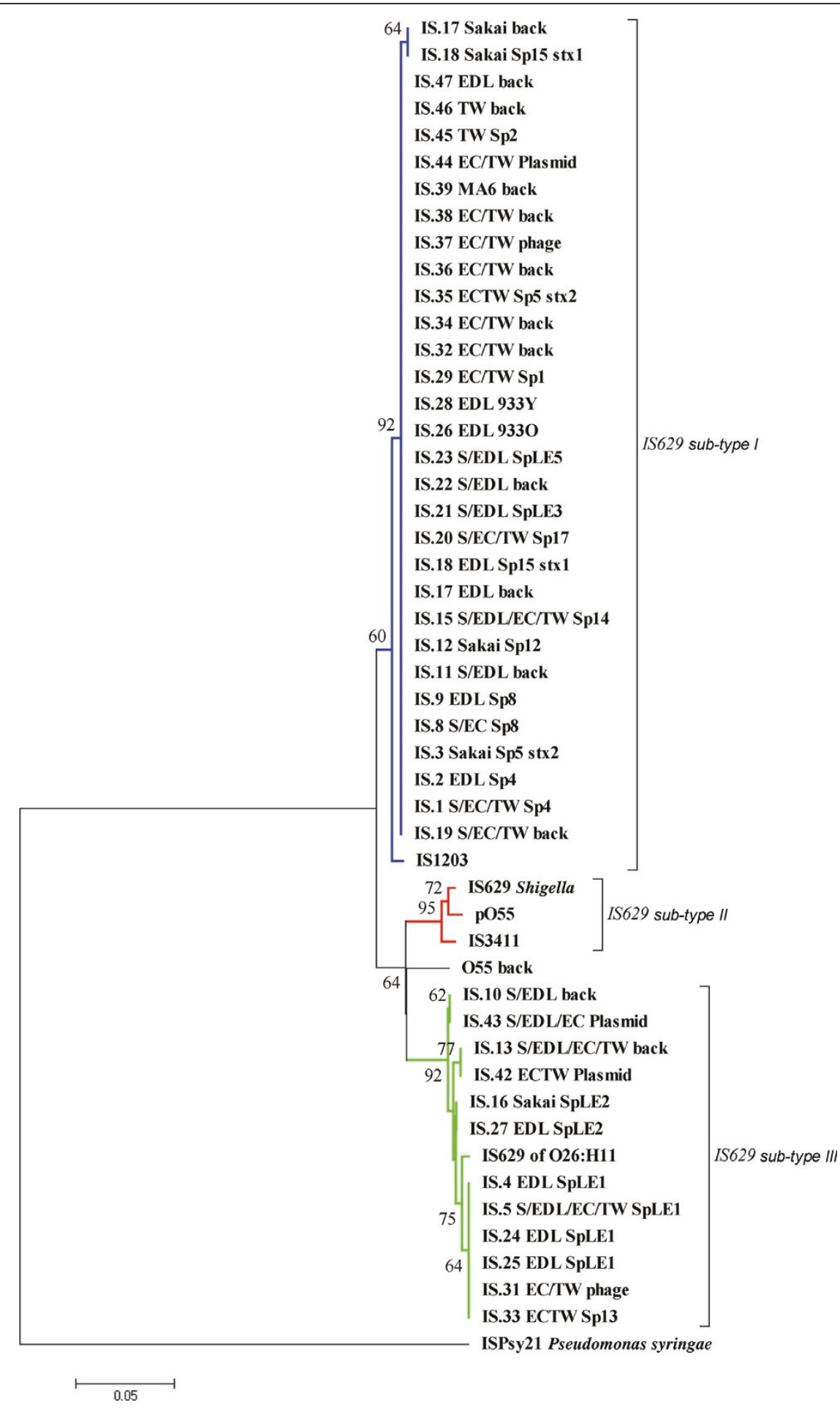

Figure 4 Phylogenetic tree of IS629 in E. coli 0157:H7 and 055:H7 showing the three different IS629 sub-types present on those five genomes. IS629 sub-type I differed from sub-type II by $4 \%$ (> 55 bp) and sub-type II differed from sub-type III by $5 \%$ (> 60 bp). IS629 sub-type II was only present in O55:H7 genome (A1/A2 CC) while sub-type I and III were present in all O157:H7 genomes (A6 CC). The evolutionary history was inferred using the Minimum Evolution method [31]. The tree is drawn to scale, with branch lengths in the same units as those of the evolutionary distances used to infer the phylogenetic tree. Bootstrap support when above 50\% is shown at nodes. Sp- prophages; SpLE prophage-like elements; and back - backbone. 
acquired by $E$. coli $\mathrm{O} 157: \mathrm{H} 7$ strains after the separation from the sub-lineage leading to the A4 CC strains therefore not carrying IS629.

\section{Discussion}

IS elements are in general regarded as genetic factors that significantly contribute to genomic diversification and evolution [7]. It was determined by Ooka et al (2009) that IS elements IS629 and ISEc8, found in the O157:H7 lineage, serve as an important driving force behind the genomic diversity. However, only a few genome-wide studies have been conducted to compare IS distributions in closely related genomes. In our study we determined that IS629 insertions in E. coli O157:H7 are widespread distributed on the genome and differ significantly from strain to strain. Although the ancestral O55: H7 strain carried only two IS629 with one on the chromosome and one on the pO55 plasmid, the four O157: H7 genomes carried between 22 and 25 IS629 copies on the chromosome and the corresponding pO157 plasmid.

IS629 does not seem to specifically integrate in sequence-based target sites, which explains the highly diverged flanking sites found in the genomes we examined. Sequence-specific insertion is exhibited to some degree by several elements and varies considerably in stringency [21]. Other elements exhibit regional preferences which are less obvious to determine [21]. IS elements frequently generate short target site duplication (TSD) flanking the IS upon insertion [21]-this feature was also observed for IS629 in the four O157:H7 strains. IS629 duplicated between 3 to 4 base pairs at the insertion site and was observed for 21 of the 47 IS629 insertion sites with matching identical base pairs up- and down-stream of IS629. A comparison of 21 TSDs created by IS629 in the four strains analyzed here did not reveal as many similarities as observed previously by Ooka et al (2009). The comparison of 25 bp up- and downstream of each insertion site did not show any similarities or patterns which would have suggested a target preference or "hot-spot" for IS629 insertions. Hence, insertion site specificity for IS629 remains unknown. However, IS629 is frequently surrounded by other IS elements ('IS islands') and was found in the same gene (gne) inserted in different sites $[4,13]$. Although no specific "hot-spot" for IS629 insertions was observed, it seems highly possible that mobile elements like plasmids, phages or phage-like elements could have functioned as vectors for IS629 introduction into O157: $\mathrm{H} 7$ genomes. These observations suggest that an insertion might occur preferentially in a region of the chromosome however these events may not be sequence specific.

IS629 insertion sites located on the backbone seem to be conserved in almost all of the strains studied here, whereby sites located on phages and phage-like areas appear to differ between all strains. These findings affirm the presence of regions of genomic stability and regions of genomic variability that exist within O157:H7 populations and closely related strains. It is noteworthy that sites associated with phages seem to be present predominantly in closely related strains. The majority of the phages present in the A6 CC strains appear to be unique to this complex. Since bacteriophages are known to contribute to the diversification of bacteria [22], they seem to be a major determinant in generating diversity among O55:H7, O157:H- and O157:H7 strains. The comparison of IS629 prevalence in A5 and A6 CC as well as IS629 insertion site prevalence in all strains allowed distinguishing strains from different complexes as it has been proposed in the evolution model for O157:H7 (Figure 1A) [11]. Adding the "same" strain from different collections, Sakai and EDL933 allowed confirmation of the stability of IS629 sites. Minimal changes in IS629 presence/absence were observed and could have occurred due to different storage conditions and passages. Despite these subtle changes, strains grouped tightly together on the parsimony tree. Therefore, this analysis can be used to further distinguish closely related $\mathrm{O} 157: \mathrm{H} 7$ strains. These findings are in agreement with a recently described IS629 analysis in three O157 lineages [23]. Similarly to what was determined for A6 and A5 CC strains, Yokoyama et al (2011) determined that IS629 distribution was biased in different $\mathrm{O} 157$ lineages, indicating the potential effectiveness of IS-printing for population genetics analysis of O157. Furthermore, Ooka et al. (2009) found that IS-printing can resolved about the same degree of diversity as PFGE. Since A1, A2 and A4 CC strains did not share IS629 insertions, their population genetics analysis however, remains limited to closely related $\mathrm{O} 157: \mathrm{H7}$ strains.

Comparison of IS629s found in O157:H7 and O55 pointed out extensive divergence between these elements. At least three different IS629 types could be distinguished differing in 55 to $60 \mathrm{bp}$. The O157:H7 strains carry IS629 elements subtype I and III whereby O55:H7 carries type II only. It is notable that only four nucleotide differences were observed among seven housekeeping genes comprising a current MLST scheme http://www.shigatox.net/ecmlst/cgi-bin/dcs between A1 CC strain DEC5A and A6 CC strain Sakai. These two strains, in particular, are taken to represent the most ancestral and most derived E. coli, respectively, in the stepwise evolutionary model for this pathogen. If the IS629 type I and III observed in A6 CC strains resulted from divergent evolution of IS629 type II, the amount of changes observed among these IS types should be similar to those observed for the MLST loci examined above. However, the number of nucleotide substitutions 
between IS629 type I and III in O157:H7 from type II in O55:H7 was 10-fold higher. Thus, the differences between IS629 types are more significant than those observed for housekeeping genes. This indicates that IS629-type II was most likely lost and IS629-type I and III were acquired independently in distinct $E$. coli O157: H7 lineages. Further supporting this thesis was the fact that one of the IS629 type II copies was found on the pO55 plasmid, which was subsequently lost during evolution towards O157:H7 strains. The other IS629 copy in $\mathrm{O} 55$, with a unique internal deletion, is located in the chromosome and appears to be part of a mobile region [24] which is absent in O157:H7 strains.

Interestingly, the ancestral IS629-deficient A2 O55:H7 strain 3256-97 is also lacking both IS629 associated regions found in the O55:H7 strains. Our analysis of common IS629 target sites demonstrated that strain 3256-97 seems to be more closely related to A4 and A5 $\mathrm{CC}$ strains than other A1 and A2 strains. Therefore, it is likely that IS629 has been lost in strain 3256-97 as well as in the hypothetical A3 precursor. These results may indicate that strain 3256-97 or a similar strain lacking IS629 might have given rise to IS629-deficient A4 CC strains.

E. coli $\mathrm{O} 157: \mathrm{H} 7$ strains carry multiple IS629 copies while the non-pathogenic K-12 strain lacks IS629 but carries other IS elements. Other pathogenic E. coli strains, amongst the top six non-O157 STEC O26:H11, O111:H- and O103:H2 [25], also harbor various copies of IS629 elements in their genomes. Genome sequences for the other three most important pathogenic nonO157 STEC; O45, O145, and O121 are not available to date thus the presence of IS629 elements is unknown. Interestingly, they also share the same reservoir with O157:H7 (e.g. cattle), shiga-toxins, haemolysin gene cluster, other virulence factors and several phages and phage-like elements [25]. Ooka et al (2009) postulated that IS-related genomic rearrangements may have significantly altered virulence and other phenotypes in O157 strains. These findings suggest that IS629 might not only have a great impact in their genomic evolution but might increase the pathogenicity of those strains as well.

\section{Conclusions}

The genomic sequence analysis showed that IS629 insertion sites exhibited a highly biased distribution. IS629 was much more frequently located on phages or prophage-like elements than in the well-conserved backbone structure, which is consistent with the observations by Ooka et al (2009). IS629 was found to be present in the A1 and one of two A2 CC strains examined as well as in all the O157:H7 strains of A5 and A6 CC, however it was totally absent in the 6 examined SFO157 strains of A4 CC. The A4 CC strains are related to but on a divergent evolution pathway from O157:H7. These results suggest that the absence of IS629 in A4 strains probably occurred during the divergence, but it is uncertain if it contributed to the divergence. Overall, IS629 had great impact on the genomic diversification of the E. coli O157:H7 lineage and might have contributed in the emergence of the highly pathogenic O157:H7.

\section{Methods}

\section{Bacterial strains}

The bacterial strains used in this study are listed in Table 2 and were chosen to represent typical EHEC and EPEC strains from the different clonal complexes from the evolution model for E. coli $\mathrm{O} 157: \mathrm{H} 7$ [11] with different serotypes (O157:H7, O157:H- and O55:H7) and different characteristics (e.g. $\beta$-glucuronidase activity (GUD), sorbitol fermentation (SOR).

\section{"In silico" analysis}

Various E. coli O157:H7 and non-O157 chromosomes and pO157 plasmids (Additional file 2, Table S1) deposited at the National Center for Biotechnology Information (NCBI) database were queried for IS629 (accession number X51586) presence and insertion loci using BLAST analysis. Furthermore, approximately 400 bp upand downstream of the flanking regions of each new localized IS629 in the chromosome and the plasmids were compared with each other. We investigated whether an IS629 was also present in the other strains or appears exclusively in either the chromosome or the plasmids.

\section{Nucleic acid extraction and determination of IS629 presence}

DNA used as the template for PCR was prepared from overnight cultures grown in Luria-Bertani Broth (LB) and purified using the MASTER PURETM DNA Purification kit (EpiCentre, Madison, WI). For determining IS629 presence in the E. coli strains, we conducted a "touchdown" multiplex PCR using IS629-specific primers targeting conserved regions of the insertion element previously described by Ooka et al. (2009): IS629insideF (5'- GAACGTCAGCGTCTGAAAGAGC-3') and IS629-insideR (5' - GTACTCCCTGTTGATGCCAG-3') and specific 16S rDNA primers: SRM86 (5'- AGAAGCACCGGCTAACTC -3') [7] and SRM87 (5'- CGCATTTCACCGCTACAC-3') [26]. The latter were used as internal amplification control. PCR amplifications were performed using $0.5 \mathrm{ng}$ of template DNA and in a final volume of $30 \mu \mathrm{l}$. The PCR reaction mixture contained 2.5 $\mathrm{U}$ of HotStart Taq Polymerase (Qiagen, Valencia, CA), 1X Taq polymerase buffer, 2.0-3.5 $\mathrm{mM} \mathrm{MgCl} 2,400 \mu \mathrm{M}$ each deoxynucleoside triphosphate (dNTP), $300 \mathrm{nM}$ each IS629 primer pair, and $300 \mathrm{nM}$ each $16 \mathrm{~S}$ rDNA primer pair. The "touchdown" PCR [27] conditions were: 1 cycle of $95^{\circ} \mathrm{C}$ for $15 \mathrm{~min} ; 10$ cycles of $95^{\circ} \mathrm{C}$ for $30 \mathrm{~s}, 69-59^{\circ} \mathrm{C}\left(-1^{\circ} \mathrm{C} /\right.$ cycle $)$ 
for $15 \mathrm{~s}$ and $72^{\circ} \mathrm{C}$ for $1: 30 \mathrm{~min}$; followed by 35 cycles consisting of $95^{\circ} \mathrm{C}$ for $30 \mathrm{~s}, 58^{\circ} \mathrm{C}$ for $20 \mathrm{~s}$, and $72^{\circ} \mathrm{C}$ for 1.5 min, and a final extension at $72^{\circ} \mathrm{C}$ for $4 \mathrm{~min}$. Amplicons were visualized on a $1 \%$ agarose gel in Tris-Borate EDTA (TBE) buffer containing $0.3 \mu \mathrm{g} / \mathrm{ml}$ ethidium bromide.

\section{Determination of IS629 specific location and IS629 insertion sites}

For the analysis of the IS629 insertion sites, primers were designed to target the different IS629 flanking regions in each strain and the plasmids. The presence/ absence of amplicons would determine the presence/ absence of the specific insertion sites and the sizes of each amplicons would indicate the presence/absence of IS629 at those loci. Potential primers were analyzed for their ability to produce stable base pairing with the template using the NetPrimer software (PREMIER Biosoft International http://www.premierbiosoft.com/netprimer/ netprlaunch/netprlaunch.html). The size of the PCR products were between $1,500-2,500 \mathrm{bp}$ in the case of IS629 presence in a strain or between $200-800$ bp in the case that the specific flanking region existed in the chromosome but did not contain an IS629 element. Each multiplex PCR contained a set of $16 \mathrm{~S}$ rDNA primers as PCR internal control (either set SRM86/SRM87 or VMP5 (5'-AGAAGCACCGGCTAACTC-3') and VMP6 (5'-CGCATTTCACCGCTACAC-3') [28]), and IS629 insertion site specific primers. The list of the 40 primer combinations for each IS629 site and PCR conditions can be found in Additional file 5, Table S4.

\section{IS629 presence/absence parsimony tree analysis}

IS629 PCR fragments sizes indicating IS629 presence/ absence and IS629 target site presence/absence identified by PCR using primers specific for each IS629 observed in 4 E. coli O157:H7 genomes were entered as binary characters (+ or -) into BioNumerics version 6.0 (Applied Maths, Saint-Martens-Latem, Belgium). IS629 presence/ absence and IS629 target site presence/absence were used to create a phylogenetic parsimony tree rooted to A5 CC strains for A5/A6 CC strains analysis (Figure 1B) and statistical support of the nodes was assessed by 1000 bootstrap re-sampling. IS629 target site presence/absence were used to create a phylogenetic parsimony tree rooted to A1/A2 CC strains for strains of the entire model (A1 A6) (Figure 1C) and statistical support of the nodes was assessed by 1000 bootstrap re-sampling.

\section{IS629 phylogenetic analysis}

Minimum evolution tree for IS629 sequences present in 4 E. coli O157:H7 genomes, two IS629 in O55:H7 genome, IS629 sequences from Shigella, two other IS629 isoforms (IS1203 and IS3411), and ISPsy21 (a member of the IS3 family and sharing only $68 \%$ homology with IS629) as out-group (Pseudomonas syringae pv. savastanoi TK2009-5) was constructed using Mega version 4.0 [29]. The evolutionary distances were computed using the Kimura 2-parameter method [30] and are in the units of the number of base substitutions per site. All positions containing gaps and missing data were eliminated from the dataset (Complete deletion option). There were a total of 299 positions in the final dataset. The statistical support of the nodes in the ME tree was assessed by 1000 bootstrap re-sampling.

\section{Additional material}

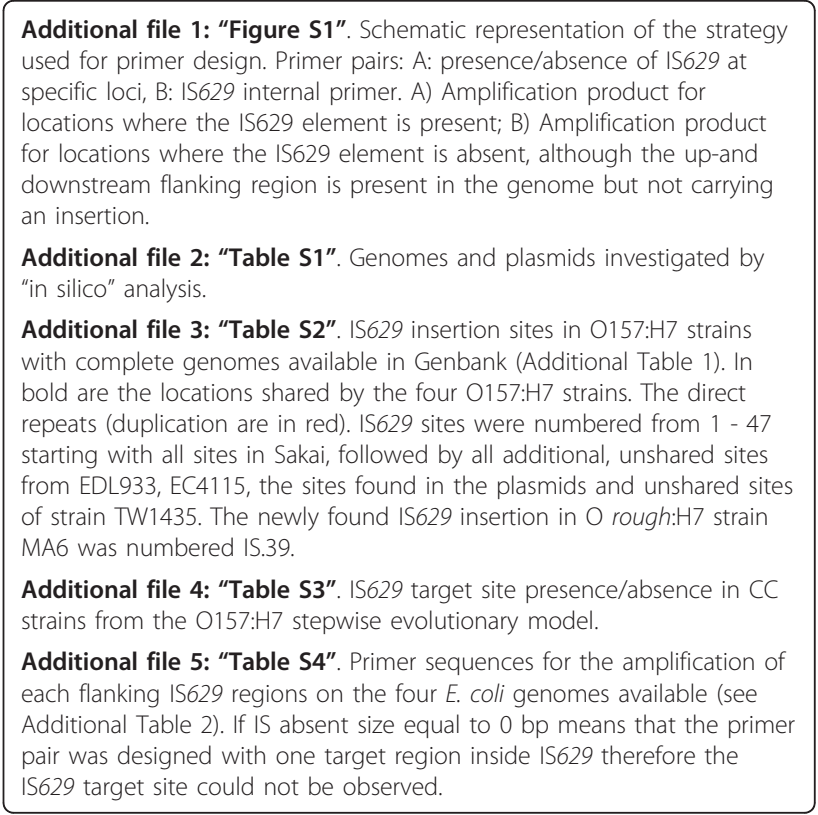

\section{Acknowledgements and Funding}

The authors thank Eric W. Brown for his helpful comments. This project was supported by an appointment to LVR through the Research Fellowship Program for the Center for Food Safety and Applied Nutrition administered by the Oak Ridge Associated Universities through a contract with the FDA.

\section{Author details}

${ }^{1}$ Division of Microbiology, Center for Food Safety and Applied Nutrition, Food and Drug Administration, 5100 Paint Branch Parkway, College Park, MD 20740, USA. ${ }^{2}$ Institute of Food Chemistry, University of Hamburg, Hamburg, Germany.

\section{Authors' contributions}

LVR conceived the study, participated in the experimental design, performed all the experiments, and participated in the production of the draft of the manuscript. MF participated in the experimental design, and production of the draft of the manuscript. NGE participated in the experimental design and coordination, performed most of the sequence analysis and phylogeny, and participated in production of the draft of the manuscript. All authors have read and approved the final manuscript.

Received: 17 December 2010 Accepted: 14 June 2011

Published: 14 June 2011 
References

1. Feng P: Escherichia coli serotype 0157:H7: novel vehicles of infection and emergence of phenotypic variants. Emerg Infect Dis 1995, 1:47-52.

2. Griffin PM, Tauxe RV: The epidemiology of infections caused by Escherichia coli 0157:H7, other enterohemorrhagic E. coli, and the associated hemolytic uremic syndrome. Epidemiol Rev 1991, 13:60-98.

3. Monday SR, Minnich SA, Feng PC: A 12-base-pair deletion in the flagellar master control gene flhC causes nonmotility of the pathogenic German sorbitol-fermenting Escherichia coli 0157:H- strains. J Bacteriol 2004, 186:2319-2327.

4. Rump LV, Feng PC, Fischer M, Monday SR: Genetic analysis for the lack of expression of the 0157 antigen in an O Rough:H7 Escherichia coli strain. Appl Environ Microbiol 2010, 76:945-947.

5. Scallan E, Hoekstra RM, Angulo FJ, Tauxe RV, Widdowson MA, Roy SL, Jones $\lrcorner$, Griffin PM: Foodborne illness acquired in the United Statesmajor pathogens. Emerg Infect Dis 2011, 17:7-15.

6. Feng P, Sandlin RC, Park CH, Wilson RA, Nishibuchi M: Identification of a rough strain of Escherichia coli 0157:H7 that produces no detectable 0157 antigen. J Clin Microbiol 1998, 36:2339-2341.

7. Ooka T, Ogura Y, Asadulghani M, Ohnishi M, Nakayama K, Terajima J, Watanabe $\mathrm{H}$, Hayashi T: Inference of the impact of insertion sequence (IS) elements on bacterial genome diversification through analysis of smallsize structural polymorphisms in Escherichia coli 0157 genomes. Genome Res 2009, 19:1809-1816.

8. Arbeit RD: Laboratory procedures for the epidemiologic analysis of microorganisms. In Manual of clinical microbiology.. 6 edition. Edited by: Murray PJ, Baron EJ, Pfaller MA, Tenover FC, Yolken RH. Washington, D.C.: ASM Press; 1995:190-208.

9. Whittam TS, Wolfe ML, Wachsmuth IK, Orskov F, Orskov I, Wilson RA: Clonal relationships among Escherichia coli strains that cause hemorrhagic colitis and infantile diarrhea. Infect Immun 1993, 61:1619-1629.

10. Feng P, Lampel KA, Karch H, Whittam TS: Genotypic and phenotypic changes in the emergence of Escherichia coli 0157:H7. J Infect Dis 1998, 177:1750-1753

11. Feng PC, Monday SR, Lacher DW, Allison L, Siitonen A, Keys C, Eklund M, Nagano H, Karch H, Keen J, Whittam TS: Genetic diversity among clonal lineages within Escherichia coli 0157:H7 stepwise evolutionary model. Emerg Infect Dis 2007, 13:1701-1706.

12. Wick LM, Qi W, Lacher DW, Whittam TS: Evolution of genomic content in the stepwise emergence of Escherichia coli 0157:H7. J Bacteriol 2005, 187:1783-1791.

13. Rump LV, Beutin L, Fischer M, Feng PC: Characterization of a gne::IS629 O rough:H7 Escherichia coli strain from a hemorrhagic colitis patient. App/ Environ Microbiol 2010, 76:5290-5291.

14. Zhou Z, Li X, Liu B, Beutin $L$, Xu J, Ren Y, Feng L, Lan R, Reeves PR, Wang L: Derivation of Escherichia coli 0157:H7 from its $055: \mathrm{H} 7$ precursor. PLoS One 2010, 5:e8700.

15. Hayashi T, Makino K, Ohnishi M, Kurokawa K, Ishii K, Yokoyama K, Han CG, Ohtsubo E, Nakayama K, Murata T, Tanaka M, Tobe T, lida T, Takami H, Honda T, Sasakawa C, Ogasawara N, Yasunaga T, Kuhara S, Shiba T, Hattori M, Shinagawa $\mathrm{H}$ : Complete genome sequence of enterohemorrhagic Escherichia coli 0157:H7 and genomic comparison with a laboratory strain K-12. DNA Res 2001, 8:11-22.

16. Ooka T, Terajima J, Kusumoto $M$, Iguchi A, Kurokawa K, Ogura Y Asadulghani M, Nakayama K, Murase K, Ohnishi M, lyoda S, Watanabe H, Hayashi T: Development of a multiplex PCR-based rapid typing method for enterohemorrhagic Escherichia coli 0157 strains. J Clin Microbiol 2009, 47:2888-2894.

17. Rump LV, Strain EA, Cao G, Allard MW, Fischer M, Brown EW, GonzalezEscalona N: Draft Genome Sequences of Six Escherichia coli Isolates from the Stepwise Model of Emergence of Escherichia coli 0157:H7. J Bacteriol 2011, 193:2058-2059.

18. Paton AW, Paton JC: Characterization of IS1203, an insertion sequence in Escherichia coli 0111:H-. Gene 1994, 150:67-70.

19. Matsutani S, Ohtsubo E: Complete sequence of IS629. Nucleic Acids Res 1990, 18:1899.

20. Zhang W, Mellmann A, Sonntag AK, Wieler L, Bielaszewska M, Tschape $H$, Karch $\mathrm{H}$, Friedrich AW: Structural and functional differences between disease-associated genes of enterohaemorrhagic Escherichia coli 0111. Int J Med Microbiol 2007, 297:17-26.
21. Mahillon J, Chandler M: Insertion sequences. Microbiol Mol Biol Rev 1998, 62:725-774.

22. Ohnishi M, Kurokawa K, Hayashi T: Diversification of Escherichia coli genomes: are bacteriophages the major contributors? Trends Microbiol 2001, 9:481-485.

23. Yokoyama E, Hashimoto R, Etoh $Y$, Ichihara S, Horikawa $K$, Uchimura M: Biased distribution of IS629 among strains in different lineages of enterohemorrhagic Escherichia coli serovar 0157. Infect Genet Evol 2010.

24. Brunder $\mathrm{W}$, Karch $\mathrm{H}$, Schmidt $\mathrm{H}$ : Complete sequence of the large virulence plasmid pSFO157 of the sorbitol-fermenting enterohemorrhagic Escherichia coli 0157:H- strain 3072/96. Int J Med Microbiol 2006, 296:467-474.

25. Fey PD, Wickert RS, Rupp ME, Safranek TJ, Hinrichs SH: Prevalence of nonO157:H7 shiga toxin-producing Escherichia coli in diarrheal stool samples from Nebraska. Emerg Infect Dis 2000, 6:530-533.

26. Monday SR, Minnich SA, Feng PC: A 12-base-pair deletion in the flagellar master control gene flhC causes nonmotility of the pathogenic German sorbitol-fermenting Escherichia coli 0157:H- strains. J Bacteriol 2004, 186:2319-2327.

27. Sambrook J, Russell RG: Molecular Cloning. A Laboratory Manual. Cold Spring Harbor: Cold Spring Harbor Laboratory Press; 2001

28. Monday SR, Beisaw A, Feng PC: Identification of Shiga toxigenic Escherichia coli seropathotypes A and B by multiplex PCR. Mol Cell Probes 2007, 21:308-311.

29. Tamura K, Dudley J, Nei M, Kumar S: MEGA4: Molecular Evolutionary Genetics Analysis (MEGA) software version 4.0. Mol Biol Evol 2007, 24:1596-1599.

30. Kimura $\mathrm{M}$ : A simple method for estimating evolutionary rates of base substitutions through comparative studies of nucleotide sequences. J Mol Evol 1980, 16:111-120.

31. Rzhetsky A, Nei M: Statistical properties of the ordinary least-squares, generalized least-squares, and minimum-evolution methods of phylogenetic inference. J Mol Evol 1992, 35:367-375.

32. Nagano $H$, Hirochi T, Fujita K, Wakamori $Y$, Takeshi K, Yano S: Phenotypic and genotypic characterization of beta-D-glucuronidase-positive Shiga toxin-producing Escherichia coli 0157:H7 isolates from deer. J Med Microbiol 2004, 53:1037-1043.

33. Nagano $H$, Okui $T$, Fujiwara $O$, Uchiyama $Y$, Tamate $N$, Kumada $H$, Morimoto Y, Yano S: Clonal structure of Shiga toxin (Stx)-producing and beta-D-glucuronidase-positive Escherichia coli 0157:H7 strains isolated from outbreaks and sporadic cases in Hokkaido, Japan. J Med Microbiol 2002, 51:405-416.

34. Eklund M, Bielaszewska M, Nakari UM, Karch H, Siitonen A: Molecular and phenotypic profiling of sorbitol-fermenting Escherichia coli 0157:Hhuman isolates from Finland. Clin Microbiol Infect 2006, 12:634-641.

doi:10.1186/1471-2180-11-133

Cite this article as: Rump et al:: Prevalence, distribution and evolutionary significance of the IS629 insertion element in the stepwise emergence of Escherichia coli O157:H7. BMC Microbiology 2011 11:133.

\section{Submit your next manuscript to BioMed Central and take full advantage of:}

- Convenient online submission

- Thorough peer review

- No space constraints or color figure charges

- Immediate publication on acceptance

- Inclusion in PubMed, CAS, Scopus and Google Scholar

- Research which is freely available for redistribution

Submit your manuscript at www.biomedcentral com/submit
C Biomed Central 